\title{
A Summary of Experimental Results on Molecular Hydrogen Formation on Dust Grain Analogues
}

\author{
Gianfranco Vidali ${ }^{1} \dagger$, J. E. Roser ${ }^{1}$, G. Manicó ${ }^{2}$, \\ and V. Pirronello ${ }^{2}$ \\ ${ }^{1}$ Syracuse University, 201 Physics Bldg., Syracuse, NY 13244-1130 USA \\ email: gvidali@syr.edu, jeroser@syr.edu \\ ${ }^{2}$ DMFCI, University of Catania \\ Viale A.Doria 6, Catania (Sicily, Italy) \\ email: gmanico@dmfci.unict.it,pirronello@dmfci.unict.it
}

\begin{abstract}
We review the main laboratory results of investigations of processes of molecular hydrogen formation on surfaces. The problem of the formation of molecular hydrogen is a fundamental issue in astrophysics/astrochemistry, because of the great importance that molecular hydrogen has for the structure and evolution of our Universe. Such experiments are done using ultra-high vacuum, low temperature, and atomic/molecular beam techniques to study the formation of molecular hydrogen on dust grain analogues in conditions as close as technically feasible to the ones present in relevant ISM environments. In experiments conducted at Syracuse University, we studied $\mathrm{H}_{2}$ formation on the three most ISM-relevant classes of surfaces: silicates, carbonaceous materials and amorphous water ice. Our experimental investigations range from the evaluation of the catalytic efficiency of the studied surfaces to the energetics of the reaction, i.e. the partition of the formation energy between the grain and the nascent molecule. Such measurements have been done by changing various parameters such as: the temperature of the interstellar dust analogue, the kinetic temperature of the atoms, the morphology of the surface and, to be completed soon, the composition of the solid. Quantitative and qualitative information on the processes of $\mathrm{H}_{2}$ formation is then fed in theoretical models to extract results that pertain to desired ISM environments.
\end{abstract}

Keywords. astrochemistry — dust — ISM: molecules — methods: laboratory

\section{Introduction}

The problem of the formation of molecular hydrogen is a fundamental issue in astrophysics/astrochemistry because of the great importance that molecular hydrogen has for the structure and evolution of the Universe. Even though great advances have taken place in the last decade from both the experimental and theoretical standpoint, this problem is not yet fully understood.

Molecular hydrogen is the most abundant molecule in space. However, since ultraviolet photons, cosmic rays, shocks and chemical reactions continuously destroy this molecule, there must be an efficient way to form it in a variety of ISM (interstellar medium) environments (Herbst 2001). As was pointed out four decades ago by Gould \& Salpeter (1963), gas-phase formation of molecular hydrogen by two-body association is extraordinarily inefficient because strongly forbidden, except in those environments - such as the early Universe - where there are enough charged particles for appreciable rates of reactions of the type: $H+e \rightarrow H^{-}+h \nu$ and $H^{-}+H \rightarrow H_{2}+e$. Hollenbach \& Salpeter (1970, 1971a,

\section{$\dagger$ Corresponding author}


1971b) in a series of papers, laid out a theory for the formation of molecular hydrogen. The formation occurs on the surface of dust grains, with a rate $R\left(\mathrm{~cm}^{-3} \mathrm{sec}^{-1}\right)$ for $\mathrm{H}+\mathrm{H} \rightarrow \mathrm{H}_{2}$ given by:

$$
R_{\mathrm{H}_{2}}=\frac{1}{2} n_{\mathrm{H}} v_{\mathrm{H}} \sigma \xi \eta n_{\mathrm{g}} .
$$

In this formula, $n_{\mathrm{H}}$ is the number density of hydrogen atoms, $v_{\mathrm{H}}$ their speed, $\sigma$ the cross section of the grain, $\xi$ the sticking coefficient, $\eta$ the probability of bond formation once two atoms interact and $n_{\mathrm{g}}$ is the number density of dust grains in the ISM. For the sticking coefficient, they used the result of their calculation of the interaction of a hydrogen atom with an ice surface, $\xi=0.3$. Assuming the probability of recombination of hydrogen atoms when on the surface to be $\eta=1$, they found that they could recover the deduced rate.

In their calculations, Salpeter and others assumed that the surface of a dust grain was covered with polycrystalline ice. The diffusion of $\mathrm{H}$ atoms on such surfaces turned out to be very fast due to tunneling. Indeed, for an average grain of $0.1 \mu \mathrm{m}$, if two hydrogen atoms are present, they calculated that the $\mathrm{H}$ atoms would find each other before evaporating.

Smoluchowski $(1981,1983)$ considered the problem of hydrogen atoms diffusing on an amorphous ice surface. Since tunneling is very sensitive, on an atomic scale, to the details of the environment through which a hydrogen atom diffuses, it is not surprising that in an amorphous medium the mobility is greatly reduced. Unfortunately, his calculations gave the result that the mobility of hydrogen atoms on an ice surface would be much too small to yield hydrogen molecules with the rate necessary to match observations.

With the availability of ultra-high vacuum techniques beginning in the late seventies, great strides were made in the study and understanding of properties of and processes at pristine surfaces. Since then, different mechanisms of reaction at surfaces have been identified. For example, in the Langmuir-Hinshelwood reaction, the partners of the reaction on a surface go through the following steps: sticking to the surface, thermalization, diffusion on the surface, formation of a bond between two reactants, and (possible) ejection of the product-molecule from the surface. This was thought to be the predominant mechanism for reactions at surfaces until recently. Alternatively, and the experimental verification of such mechanism has been realized only recently, an incoming atom can directly react with a partner on the surface without prior accommodation on it (this is called the Eley-Rideal mechanism). Another mechanism, the hot-atom mechanism, calls for the incoming atom to retain on the surface part of its original kinetic energy (or to gain some energy from the surface) in order to move across it at superthermal speed. For a review of such mechanisms as applied to reactions of interest in the ISM, see Vidali et al. (2005) and references cited therein.

The strength of the bond between an adatom (i.e., an atom adsorbed on a surface) and the surface itself is of course of primary importance for reactions to proceed. In typical diffuse cloud ISM conditions, the dust grain is bare with few $\mathrm{H}$ atoms on it; thus, in this case, the Eley-Rideal mechanism doesn't seem to be the dominating process because the chance of an atom from the gas-phase to hit another on the surface is slim. Therefore, an incoming $\mathrm{H}$ atom must be able to diffuse on the surface in order to find another $\mathrm{H}$ atom and react with it. Assuming that the dust grain temperature is in the $15-20 \mathrm{~K}$ range, and that the grain (a silicate or a carbonaceous grain, or a composite particle of the two) is amorphous - as per observations (see Whittet 2003 for a review), an $\mathrm{H}$ atom will diffuse on the surface of the grain only if it is held by weak binding forces. In the end, successful production of molecular hydrogen in ISM conditions will occur when the H-surface bond 
strength is weak enough to allow mobility and strong enough to keep the H atom long enough on the surface to find another partner (the activation energy barrier for diffusion is roughly one third of the binding site energy for an atomically ordered surface, but can vary considerably due to chemical bonding and morphology). It is expected that chemical and morphological heterogeneity increase the chance of successful reactions by providing a rich landscape of energy sites where the atoms can reside long enough.

In the process of the formation of a molecule, energy is released. How this energy is partitioned between the molecule and the solid, and within the molecule, is of obvious astrophysical importance, both because the kinetic energy of $\mathrm{H}_{2}$ can contribute to the energy budget of the cloud, and because there is the possibility of observing the formation of molecules on actual dust grains through the detection of vibrationally hot molecules in the ISM.

At the end of the '90s, we began a laboratory program to investigate how these competing processes would play out in the formation of $\mathrm{H}_{2}$ under ISM conditions; we also wanted to learn what kind of dust grain surfaces would be most efficient in the formation of molecular hydrogen. A study of the formation of $\mathrm{H}_{2}$ should also give us clues on the formation of more complex molecules on dust grain surfaces via hydrogenation reactions.

The first experiments were done on the polished surface of polycrystalline olivine (Pirronello et al. 1997a; Pirronello et al. 1997b.) They were followed by measurements of $\mathrm{H}_{2}$ formation on amorphous carbon (Pironello et al. 1999), and amorphous water-ice layers (Manicó et al. 2001; Roser et al. 2002). In 2003, Roser et al. (2003) and, independently, another group, Hornekær et al. (2003), measured the time-of-flight of molecules that were just formed on the surface and were being ejected from it. The next step, which is already under way (Perry et al. 2002), is to study the distribution of the energy of the molecule within the ro-vibrational degrees of freedom. There are efforts under way to measure the excitation of the molecule in actual ISM environments; preliminary studies have not been successful, however, in singling out excitation of the molecules that can be ascribed to molecule formation events and not to pumping mechanisms due to shocks and photon absorption (Takahashi \& Uehara 2001; Tiné et al. 2003).

In the next section, we give a brief overview of the experimental methods; the reader is referred to a recent publication (Vidali et al. 2005) for technical details. In $\S 3$, we summarize the results; a discussion of the significance of these results and how they can be applied to ISM environments is given in $\S 4$, together with comments about experiments conducted in other laboratories.

\section{Experimental Methods}

The experiments conducted in our laboratory at Syracuse University, N.Y. were designed to study the processes leading to the formation of molecular hydrogen on surfaces under conditions that come as close as technically feasible to the ones in the most relevant ISM environments. The main requirements are low $(\sim 10 \mathrm{~K})$ sample temperature, low background pressure $\left(\sim 10^{-10}\right.$ torr, equivalent to the typical number density in dense clouds), use of non contaminating materials and techniques to preserve the integrity of samples during the the experiments, and low fluxes of $\mathrm{H}$ atoms. Except for the last condition, the others can be easily met with the employment of ultra-high vacuum and standard low-temperature techniques. We used atomic beam techniques to deliver an angular resolved $\left(10^{-6}\right.$ steradian), low flux $\left(10^{12}\right.$ atoms $\left.\mathrm{sec}^{-1} \mathrm{~cm}^{-2}\right)$, low energy (200$300 \mathrm{~K}$ ) beam of $\mathrm{H}$ atoms to the sample. To avoid that the signal of successful events (i.e., the detection of $\mathrm{H}_{2}$ ) be overwhelmed by the contribution from the undissociated fraction of the beam (about $10-15 \%$ ) or background gas (mostly $\mathrm{H}_{2}$ in a UHV system), we used 
two beams simultaneously, one of $\mathrm{H}$ and the other of $\mathrm{D}$ atoms. In this case, HD can form only on the sample surface and we obtain a large gain in sensitivity.

The apparatus consists of two triply differentially pumped beam lines, each equipped with a radiofrequency dissociation source, a scattering chamber housing the sample and a mass-discriminating detector that can be rotated around it. There is also a time-of-flight appendix connected to the sample chamber and equipped with its own detector. The background pressure in the main chamber is in the $10^{-10}$ torr range and the sample can be cooled down to $\sim 4.5 \mathrm{~K}$. The experiment consists of sending $\mathrm{H}$ and $\mathrm{D}$ atoms onto the sample for a fixed amount of time (exposure); at the same time, the products of reaction are detected using a mass-discriminating detector. At the end of the exposure, the sample temperature is quickly increased to either evaporate molecules that formed on the surface, or cause the diffusion of atoms on the surface with consequent reaction and ejection from the surface. The purpose of raising the temperature of the sample, called TPD or temperature programmed desorption, is to obtain information on the kinetics of the reaction and on its yield. After much experimentation, we find that two key features are essential for a successful experiment designed to obtain useful information about similar processes taking place in the ISM: the use of two isotopes, and of low flux. Still, our flux is several orders of magnitude higher than the ones in the ISM. Therefore, models are necessary to make predictions of $\mathrm{H}_{2}$ formation utilizing laboratory data. For details, see Biham et al. (2005).

\section{Results}

Over the years, we have studied the formation of $\mathrm{H}_{2}$ on the following solids that are representative (within the technical capability at that time) of the ones present in key ISM environments: a polished polycrystalline telluric sample of olivine, an amorphous carbon sample, and amorphous water ice layers. The information that we obtained can be summarized as follow:

- There is evidence that the reaction kinetics on the polycrystalline sample is of the second order at the lowest coverage (a small fraction of a layer). This means that the recombination rate depends quadratically on the surface density of the reactants. In the TPD experiments, second order kinetics is characterized by the gradual shift of the maximum of the peak to lower temperature as the coverage is increased (Pironello et al. 1997a; Chan et al. 1978). For other samples, amorphous carbon and amorphous ice, the features of the signal are broader and it is harder to separate effects due to the morphology of the surface from the signature of the kinetics. Nonetheless, in the case of desorption from low-densisty amorphous ice, at least one peak of the differential yield $\left(\mathrm{d} N_{\mathrm{HD}} / \mathrm{d} T\right)$ of HD shows a clear indication of second order kinetics (Perets et al. 2005).

- Thermal activation is necessary to get the reaction going, indicating that tunneling alone is not sufficient to assure the necessary diffusion at these exposures and sample temperature (see Fig. 1 and the discussion below).

- The range of temperature over which there is significant $\mathrm{H}_{2}$ formation is rather narrow, and, in any case, is below $\sim 20 \mathrm{~K}$, as shown in Figure 2.

From the study of the shape and position of the desorption rate vs. time (or temperature) - see Figure 1 for an example, one can obtain, with the help of sets of rate equations, activation energy barriers for desorption and diffusion of atoms and molecules (Katz et al. 1999; Peretz et al. 2005).

Further evidence that the formation of HD is initiated by thermal energy is given in Figure 1 which compares the differential HD yield $\left(\mathrm{d} N_{\mathrm{HD}} / \mathrm{d} T\right)$, as a function of the sample temperature $\mathrm{T}$ during a TPD, obtained in two different experiments on the same 
Figure 1. TPD traces of HD desorption after irradiation with HD molecules (o) and $\mathrm{H}+\mathrm{D}$ atoms $(+)$ on amorphous low-density water ice. The solid lines are fits obtained by a rate equations model. See Perets et al. (2005) for details.

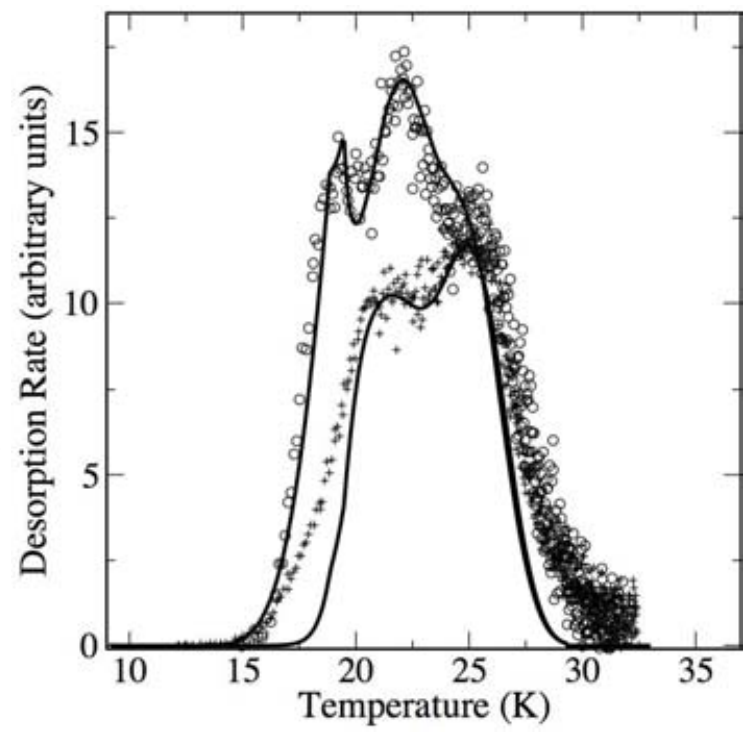

surface of low density amorphous ice (Perets et al. 2005). In one, the sample is exposed to the $\mathrm{H}$ and $\mathrm{D}$ beams and then the sample temperature is raised quickly, and the HD molecules ejected from the surface are detected. In the other, the sample is dosed with $\mathrm{HD}$ molecules and then the sample is heated as before. If $\mathrm{H}$ and $\mathrm{D}$ were very mobile and reacted quickly making $\mathrm{HD}$, then the heat pulse should give the same pattern in both experiments, since these would be experiments of desorbing HD molecules already present on the surface. On the other hand, if, in the first experiment, H and D atoms are not mobile and heat is necessary to make them diffuse and react, the desorption pattern is expected to be different, because in this case it probes the end result of three different processes: diffusion of $\mathrm{H}$ and $\mathrm{D}$, reaction and desorption of $\mathrm{HD}$; in the other, it probes the desorption of HD only. Indeed, the former is what is observed.

Experimentally, from measurements such as the ones described above, and using no assumption, one can get the recombination efficiency (defined as the probability to form a molecule for each two atoms sent onto the surface) at different sample temperature, as shown in Figure 2. To obtain it, the beam fluxes are measured first. Then TPD traces, such as those in Figure 1 are integrated to obtain the total number of molecules detected. The ratio of these two quantities (corrected for instrumental effects and for the fact that other reaction pathways are possible) gives the recombination efficiency, a number between 0 and 1 . Strictly speaking, there is also a contribution to the HD yield due to $\mathrm{H}$ and $\mathrm{D}$ atoms reacting during the time of the exposure and forming $\mathrm{HD}$ molecules that are released in the gas phase. These molecules are found to contribute little to the yield coming from the subsequent TPD experiment.

To obtain the recombination efficiency under ISM conditions, one has to obtain the probability to form $\mathrm{H}_{2}$ at ISM $\mathrm{H}$ fluxes on a grain of a given size; then one has to integrate over the distribution of grain sizes in the ISM. This procedure is described elsewhere in these proceedings (Biham et al. 2005) and by Vidali et al. (2005), Perets et al. (2005), Katz et al. (1999).

Recently, Hornekær et al. (2003) presented interesting results on $\mathrm{H}+\mathrm{D}$ recombination on porous and non-porous amorphous solid water (ASW). Their experiments on the formation of $\mathrm{HD}$ on amorphous water ice are similar to ours. Their atom beams had 
Figure 2. Recombination efficiency of molecular hydrogen vs. temperature of the sample. Filled circles are for high-density amorphous water ice, filled squares are for low-density amorphous water ice prepared by heating high-density amorphous ice. Filled triangles are for water vapor-deposited low-density amorphous ice. The scatter in the data points reflects the variability in the ice preparation methods. Empty circles are for polycrystalline olivine and empty squares for amorphous carbon. From Vidali et al. (2005). (Please note that the figure caption in Vidali et al. is incorrect.)

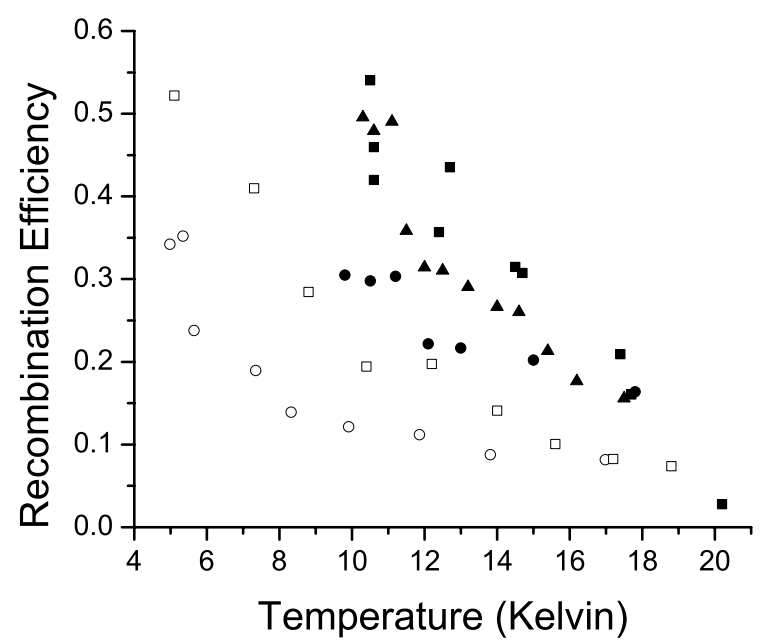

fluxes $\sim 10^{13}$ (atoms $\mathrm{cm}^{-2} \mathrm{sec}^{-1}$ ) about one order of magnitude higher than the ones used in our experiments, while the range of exposure times was comparable.

Hornekær et al. performed TPD experiments in which they irradiated H and D atoms either simultaneously or sequentially after waiting a delay time interval before dosing the other isotope. On porous ASW, their results are consistent with a recombination reaction occurring quickly during atom dosing due to the high mobility of the adsorbed atoms even at temperatures as low as $8 \mathrm{~K}$. This high mobility was attributed either to quantum mechanical diffusion or to the "hot-atom" mechanism. Thermal activation is not likely to play a significant role at this temperature. This conclusion is sensible in light of the high coverages of $\mathrm{H}$ and $\mathrm{D}$ atoms used in these experiments, which required the adsorbed atoms to diffuse only for very short distances before encountering each other. As already suggested by Pirronello et al. (2004a, 2004b), the hot-atom mechanism may be able to provide the required mobility. In this case $\mathrm{H}$ and $\mathrm{D}$ atoms retain a good fraction of their gas phase kinetic energy during the accommodation process, enabling them to travel on the ice surface and inside its pores for several tens of Angstroms (Buch \& Zhang 1991; Takahashi \& Uehara 2001), exploring several adsorption sites and recombining upon encountering atoms already adsorbed on the surface.

In our experiments, we used low fluxes of atoms and short irradiation times to have a low coverage of $\mathrm{H}$ atoms on the surface. Therefore, in our situation the $\mathrm{H}$ atom that has just landed on the surface (the "hot atom") and is bouncing around and losing energy, has a far smaller probability of finding another $\mathrm{H}$ atom on the surface than in the Hornekær experiments because of the much lower coverage in our case. This has been confirmed by doing a calculation using rate equations in which the probability of reaction to form $\mathrm{H}_{2}$ is proportional to the region of surface spanned by the hot atom. By using values of the fluxes and exposure times employed in their experiments and in ours, as well as estimates from molecular dynamics simulations of the distance traveled by an $\mathrm{H}$ atom landing on an ice surface (Buch \& Zhang 1991; Takahashi \& Uehara 2001) we do find that in our case the probability of making $\mathrm{H}_{2}$ on the cold surface without thermal activation is very small, but large in the case of Hornekær's experiment.

As observed experimentally by Hornekær et al., the molecules remain trapped in the ice until the TPD is initiated. In our experiment, the atoms are too far apart from each 
Figure 3. Recombination efficiency of molecular hydrogen vs. temperature for ISM conditions obtained using parameters from experiments. For details, see Biham, Lipshatz \& Perets (2005).

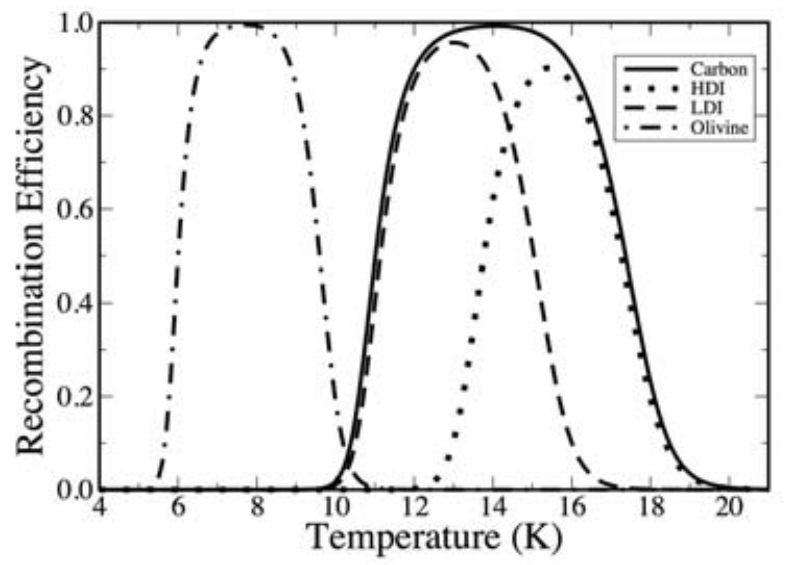

other during the deposition phase, and the theoretical analysis confirms our original interpretation (Roser et al. 2002 for $\mathrm{H}_{2}$ formation on ice; Pirronello et al. 1997a,b, and Katz et al. 1999 for $\mathrm{H}_{2}$ formation on olivine and amorphous carbon), that atoms remain confined to their sites until the TPD is initiated. At higher coverage, but still less than one layer, first order kinetics is also observed on olivine (Pirronello et al. 1997a).

Finally, we did an experiment to determine the distribution of kinetic energies of hydrogen molecules forming and desorbing from the sample surface. An amorphous water ice sample was exposed to $\mathrm{H}$ and $\mathrm{D}$ atoms; the sample was turned towards the time-offlight (t-o-f) line and a TPD was done. The molecules entering the t-o-f line were chopped by a high-speed slotted wheel. The particles emerging from the surface had MaxwellBoltzmann distribution of velocities with an average kinetic energy corresponding to a surface temperature of $\sim 24 \mathrm{~K}$. A similar result was obtained independently by Hornekær et al. (2003). The interpretation of this result is that HD forms in the porous structure of amorphous ice and the emerging HD molecule makes enough collisions leaving the surface that it loses most of its kinetic energy (Vidali et al. 2004).

\section{Discussion and Astrophysical Implications}

We have presented a summary of experimental results on $\mathrm{H}_{2}$ formation obtained by exposing atomic hydrogen to surfaces belonging to the three classes that can be considered good analogues of interstellar grains (silicates, carbonaceous particles, and ices). The data and theoretical modeling show that, depending on the experimental conditions, the L-H mechanism or the hot-atom/Eley-Rideal mechanisms are behind the formation of molecular hydrogen in several astrophysically relevant situations. Most of the experiments performed at low temperature (5-30 K) show that when physisorption sites are involved, hydrogen atoms that are accommodated on the surface recombine with high efficiency (close to 1) but only in a narrow temperature range through the L-H mechanism, the only one that can work when $\mathrm{H}$ adatoms are on the average well separated from each other and when a relatively high mobility is required for them to encounter and react. Experimental evidence (such as the detection of second order kinetics in the $\mathrm{H}+$ $\mathrm{D} \rightarrow \mathrm{HD}$ reaction and the difference between experiments done with $\mathrm{H}$ and $\mathrm{D}$ atoms and with HD molecules; see Figure 1) suggests that the mobility of adatoms that is necessary for an efficient reaction must be given by either thermal hopping or by what we call 
"thermally assisted tunneling", while the unassisted temperature-independent tunneling seems to be ruled out because experiments performed at liquid helium temperature show the need of thermal activation to obtain the mobility of adatoms (see Pirronello et al. 1997a). The drop in efficiency of this mechanism at "high temperature" (which is $\sim 20 \mathrm{~K}$ for the studied surfaces so far) is due to the increasing importance of evaporation of the adatoms, which limits the residence time on the surface.

Whenever the "coverage" of $\mathrm{H}$ adatoms is high enough, the hot-atom/Eley-Rideal mechanism becomes efficient. In this case, an atom coming from the gas phase during the accommodation process (and hence during the multiple collisions of atoms with the surface) explores several adsorption sites and when it hits an $\mathrm{H}$ adatom it reacts with it forming $\mathrm{H}_{2}$. At "high temperature" the efficiency of this mechanism also drops due to the shortening of the residence time of atoms on the surface which makes difficult to maintain a high enough coverage. Data presented by different groups on the formation of $\mathrm{H}_{2}$ on amorphous water ice surfaces are in substantial agreement, but the interpretation differs considerably. Essentially, Hornekær et al. (2003) claim that there is fast diffusion even at low surface temperature $(8 \mathrm{~K})$ with the consequence that HD forms during irradiation. We proposed (Pirronello et al. 1997a) that this fast recombination can be explained by the hot-atom mechanism. This mechanism is expected to be efficient in the case of high coverage, which is the experimental condition used by Hornekær et al. for amorphous water ice and by Pirronello et al. for the measurements on olivine at high coverage (Pirronello et al. 1997a).

The implications of the reported results in the astrophysical environments can be summarized in the following way. Formation of molecular hydrogen in dense clouds or in clumps is well explained by the $\mathrm{L}-\mathrm{H}$ mechanism, because the temperature of grains is well inside the range of values in which mobility of adatoms is high. In very cold regions (with temperature below 9-10 K) the hot-atom/Eley-Rideal mechanism allows efficient formation of molecular hydrogen, because on grains the coverage of $\mathrm{H}$ atoms reaches high values due to the lack of evaporation of adatoms and the low efficiency of the L-H mechanism in producing $\mathrm{H}_{2}$ that could leave the grain.

Ongoing experiments on amorphous olivines could verify whether the observed trend (that recombination is higher on low-temperature amorphous surfaces than on polycrystalline ones) is correct or not.

In the case of astrophysical environments where the temperature of grains is higher yet, for example in PDRs, the possibility of formation of $\mathrm{H}_{2}$ relies on the presence of chemisorption sites. Most of the discussion given above applies also in the case of chemisorption sites, both regarding the L-H mechanism (in an appropriate range of grain temperature), and the hot-atom/Eley-Rideal mechanism. The most relevant merit of chemisorption sites is the high binding energy (of the order of an $\mathrm{eV}$ ) which extends the residence time of $\mathrm{H}$ adatoms against evaporation even when the grain temperature is in the hundreds of degree K. Of course it comes with drawbacks. In this case an energy barrier to enter the chemisorption site (which could be of the order of a few tenths of eV) might be present. Such a barrier would impede low-energy atoms to chemisorb on the surface. This is not a problem in PDRs, but obviously it gives a prominent role to physisorption sites at low temperature.

In summary, the importance of dust grain surfaces in the catalytic formation of $\mathrm{H}_{2}$ has long been recognized, but only recently, beginning at the end of the 1990s, has it been possible to do experiments to better understand this important process. As data from different surfaces and under different conditions were obtained, it became clear that different mechanisms operate depending on the conditions chosen for the experiments. In the end, a clear picture has emerged. The formation of $\mathrm{H}_{2}$ at low temperature on dust 
grain analogues requires thermal activation, and is dominated by the L-H mechanism at low coverage and the hot-atom mechanism at higher coverage, close to monolayer completion. This can explain both ours and Hornekær et al.'s data. We also learnt that theoretical tools such as rate equations (see Biham et al. 2005) are necessary to extract physical parameters from the experimental data, which are then used to predict how the reaction will go under truly ISM conditions.

\section{Acknowledgments}

This work was supported by NASA through grants NAG5-9093 and NAG5-11438 (G.V) and by the Italian Ministry for University and Scientific Research through grant 21043088 (V.P). We thank A. Lipshatz and H. Perets for the preparation of Figure 3.

\section{References}

Biham, O., Lipshatz, A., \& Perets, H.B. 2005, in this volume

Buch, V. \& Zhang, Q. 1991, Ap. J. 379, 647

Chan, C.M., Aris, R., \& Weinberg, W.H. 1978, Appl.Surf.Sci. 1, 360

Gould, R. J. \& Salpeter, E. E. 1963, Ap. J. 53, 79

Herbst, E. 2001, Chem. Soc. Rev. 30, 168

Hollenbach, D. \& Salpeter, E.E. 1970, J. Chem. Phys. 53, 79

Hollenbach, D. \& Salpeter, E.E. 1971a, Ap. J. 163, 155

Hollenbach, D., Werner, M.W., \& Salpeter, E.E. 1971b, Ap. J. 163, 165

Hornekær, L., Baurichter, A., Petrunin, V.V., Field, D., \& Luntz, A.C. 2003, Science 302, 1943

Katz, N., Furman, I., Biham, O., Pirronello, V., \& Vidali, G. 1999, Ap. J. 522, 305

Manicó, G., Raguni, G., Pirronello, V., Roser, J.E., \& Vidali, G. 2001, Ap. J. 548, L253

Perets, H.B., Biham, O., Manicó, G., Pirronello, V., Roser, J., Swords, S., \& Vidali, G. 2005, Ap. J., in press

Perry, J.S.A., Gingell, J.M., Newson, K.A., To, J., Watanabe, N., \& Price, S.D. 2002, Meas. Sci. Tech. 106, 8998

Pirronello, V., Liu, C., Shen, L., \& Vidali, G. 1997a, Ap. J. 475, L69

Pirronello, V., Biham, O., Liu, C., Shen, L., \& Vidali, G. 1997b, Ap. J. 483, L131

Pirronello, V., Liu, C., Roser, J.E., \& Vidali, G. 1999, A\&A 344, 681

Pirronello, V., Manicó, G., Roser, J.E., \& Vidali, G. 2004a, in Astrophysics of Dust, ed. A. Witt et al. (ASP Series, Sheridan Books Ann Arbor, MI, USA) p. 529

Pirronello, V., Manicó, G., Roser, J.E., \& Vidali, G. 2004b, The Dense Interstellar Medium in Galaxies, ed. S. Pfalzner et al. (Springer-Verlag, Berlin) p. 525

Roser, J.E., Manicò, G., Pirronello, V., \& Vidali, G. 2002, Ap. J. 581, 276

Roser, J.E., Swords, S., Vidali, G., Manicó, G., \& Pirronello, V. 2003, Ap. J. 596, L55

Smoluchowski, R. 1981, Ap. and Space Sci. 75, 353

Smoluchowski, R. 1983, J. Phys. Chem. 87, 4229

Takahashi, J., \& Uehara, H. 2001, Ap. J. 561, 843

Tine, S., Williams, D.A., Clary, D.C., Farebrother, A.J., Fisher, A.J., \& Meijer, A.J.H.M. 2003, Ap. Space Sci. 288, 377

Vidali, G., Roser, J.E., Manicó, G., Pirronello, V., Perets, H.B., \& Biham, O. 2005, J. of Phys.: Conf. Series 6, 36

Vidali, G., Roser, J.E., Manicó, G. \& Pirronello, V. 2004, J. Geophys. Res. 109, E07S14

Whittet, D.C.B. 2003, Dust in the Galactic Environment (Cambridge University Press) 


\section{Discussion}

HorneKÆR: Since the LDA ice is also less porous (due to annealing) than the highdensity ice, a difference between the TPD spectra for HD from recombination and HD from molecular deposition could be due to the immediate desorption of newly formed HD from low-energy binding sites. The case is different for high-density, porous ices. In this case I would expect the TPD spectra from directly deposited and atomically recombined HD to be identical. Is this the case in your data?

VIDALI: As to the first part of the question, the fact that there is evidence of secondorder kinetics, plus the shape of the TPDs, strongly suggest that the formation of HD requires thermal activation. As to the second part of the question, we have not studied the high-density ice with the same detail as the low-density one. The reason is that the high-density ice is prepared at $10 \mathrm{~K}$ and the ice is never taken above $\sim 32-34 \mathrm{~K}$. We suspect that there is a dependence of the morphology of the ice on subtle changes in the preparation methods, and thus it is harder to obtain a "characteristic" high-density ice. Therefore, we concentrated most of our attention on the study of recombination processes on low-density ice.

Allamandola: These are beautiful experiments and very hard to do. Do you have an idea of what will happen if more realistic ices are used, for example containing $\mathrm{CO}$ ?

VIDALI: In the experiments we have done so far, we tried to keep it simple in order to get the physics of the processes right. It is of great interest to study more realistic ices. I suspect that the presence of $\mathrm{CO}$ would complicate matters considerably from an experimental point of view, because $\mathrm{CO}$ sublimates easily, but it would be definitely an interesting ice mixture to study. 